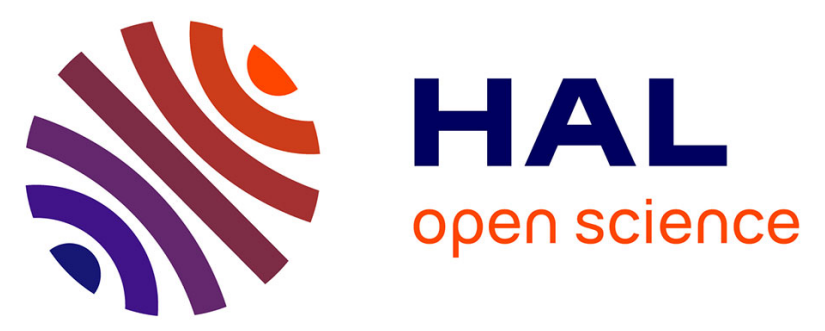

\title{
High-throughput sequencing contributes to the diagnosis of tubulopathies and familial hypercalcemia hypocalciuria in adults
}

Marguerite Hureaux, Emma Ashton, Karin Dahan, Pascal Houillier, Anne Blanchard, Catherine Cormier, Eugenie Koumakis, Daniela Iancu, Hendrica Belge, Pascale Hilbert, et al.

\section{To cite this version:}

Marguerite Hureaux, Emma Ashton, Karin Dahan, Pascal Houillier, Anne Blanchard, et al.. Highthroughput sequencing contributes to the diagnosis of tubulopathies and familial hypercalcemia hypocalciuria in adults. Kidney International, 2021, 96 (6), pp.1408-1416. hal-03116148

\author{
HAL Id: hal-03116148 \\ https://hal.science/hal-03116148
}

Submitted on 25 Jan 2021

HAL is a multi-disciplinary open access archive for the deposit and dissemination of scientific research documents, whether they are published or not. The documents may come from teaching and research institutions in France or abroad, or from public or private research centers.
L'archive ouverte pluridisciplinaire HAL, est destinée au dépôt et à la diffusion de documents scientifiques de niveau recherche, publiés ou non, émanant des établissements d'enseignement et de recherche français ou étrangers, des laboratoires publics ou privés.

\section{(ㅇ)(1) $\$$}

Distributed under a Creative Commons Attribution - NonCommerciall 4.0 International 


\title{
High-throughput sequencing contributes to the diagnosis of tubulopathies and familial hypercalcemia hypocalciuria in adults
}

\author{
Marguerite Hureaux ${ }^{1,2}$, Emma Ashton ${ }^{3}$, Karin Dahan ${ }^{4,5}$, Pascal Houillier ${ }^{2,6}$, Anne Blanchard ${ }^{2,7}$, \\ Catherine Cormier $^{8}$, Eugenie Koumakis ${ }^{8}$, Daniela lancu ${ }^{9}$, Hendrica Belge ${ }^{4}$, Pascale Hilbert ${ }^{4}$, \\ Annelies Rotthier ${ }^{10}$, Jurgen Del Favero ${ }^{10}$, Franz Schaefer ${ }^{11}$, Robert Kleta ${ }^{9,12}$, Detlef Bockenhauer ${ }^{9,12}$, \\ Xavier Jeunemaitre ${ }^{1,2}$, Olivier Devuyst ${ }^{5,13}$, Stephen B. Walsh ${ }^{9}$ and Rosa Vargas-Poussou ${ }^{1}$
}

${ }^{1}$ Department of Genetics, Assistance Publique Hôpitaux de Paris, Hôpital Européen Georges-Pompidou, Paris, France; ${ }^{2}$ Paris Descartes Université, Sorbonne Paris Cité, Paris, France; ${ }^{3}$ North East Thames Regional Genetics Service Laboratories, Great Ormond Street Hospital for Children National Health Service Foundation Trust, London, UK; ${ }^{4}$ Center of Human Genetics, Institut de Pathologie et Génétique, Gosselies, Belgium; ${ }^{5}$ Division of Nephrology, Université Catholique de Louvain Medical School, Brussels, Belgium; ${ }^{6}$ Department of Physiological Functional Investigations, Assistance Publique Hôpitaux de Paris, Hôpital Européen Georges-Pompidou, Paris, France; ${ }^{7}$ Assistance Publique Hôpitaux des Hôpitaux de Paris, Hôpital Européen Georges-Pompidou, Centre d'Investigation Clinique 1418, Paris, France; ${ }^{8}$ Rheumatology Department, Assistance Publique Hôpitaux de Paris, Hôpital Cochin, Reference Center for Rare Calcium and Phosphate Diseases, Paris, France; ${ }^{9}$ Department of Renal Medicine, University College London, London, UK; ${ }^{10}$ Multiplicom N.V. (a part of Agilent Technologies), Niel, Belgium; ${ }^{11}$ Paediatric Nephrology, Heidelberg University Center for Pediatrics and Adolescent Medicine, Heidelberg, Germany; ${ }^{12}$ Department of Pediatric Nephrology, Great Ormond Street Hospital for Children National Health Service Foundation Trust, London, UK; and ${ }^{13}$ Institute of Physiology, Zurich Center for Integrative Human Physiology (ZIHP), Mechanisms of Inherited Kidney Disorders Group, University of Zurich, Zurich, Switzerland

Hereditary tubulopathies are rare diseases with unknown prevalence in adults. Often diagnosed in childhood, hereditary tubulopathies can nevertheless be evoked in adults. Precise diagnosis can be difficult or delayed due to insidious development of symptoms, comorbidities and polypharmacy. Here we evaluated the diagnostic value of a specific panel of known genes implicated in tubulopathies in adult patients and compared to our data obtained in children. To do this we analyzed 1033 non-related adult patients of which 744 had a clinical diagnosis of tubulopathy and $\mathbf{2 8 9}$ had a diagnosis of familial hypercalcemia with hypocalciuria recruited by three European reference centers. Three-quarters of our tubulopathies cohort included individuals with clinical suspicion of Gitelman syndrome, kidney hypophosphatemia and kidney tubular acidosis. We detected pathogenic variants in 26 different genes confirming a genetic diagnosis of tubulopathy in $29 \%$ of cases. In 16 cases $(2.1 \%)$ the genetic testing changed the clinical diagnosis. The diagnosis of familial hypercalcemia

\footnotetext{
Correspondence: Rosa Vargas-Poussou, Hôpital Européen Georges-Pompidou, Department of Molecular Genetics, 20-40 rue Leblanc, 75015 Paris, France. E-mail: rosa.vargas@aphp.fr; or Stephen B. Walsh, Department of Renal Medicine, University College London, Pond Street, Hampstead, London NW3 2QG, UK. E-mail: stephen.walsh@ucl.ac.uk; or Olivier Devuyst, University of Zurich, Institute of Physiology, Zurich Center for Integrative Human Physiology (ZIHP), Mechanisms of Inherited Kidney Disorders Group, Winterthurerstr. 190, CH-8057 Zürich, Switzerland. E-mail: olivier.devuyst@uclouvain.be or olivier. devuyst@uzh.ch
}

Received 19 June 2019; revised 7 August 2019; accepted 22 August 2019; published online 16 September 2019 with hypocalciuria was confirmed in $12 \%$ of cases. Thus, our work demonstrates the genetic origin of tubulopathies in one out of three adult patients, half of the rate observed in children. Hence, establishing a precise diagnosis is crucial for patients, in order to guide care, to survey and prevent chronic complications, and for genetic counselling. At the same time, this work enhances our understanding of complex phenotypes and enriches the database with the causal variants described.

Kidney International (2019) 96, 1408-1416; https://doi.org/10.1016/ j.kint.2019.08.027

KEYWORDS: adults; genetic testing; next-generation sequencing; tubulopathy

Crown Copyright (c) 2019, Published by Elsevier, Inc., on behalf of the International Society of Nephrology. All rights reserved.

nherited renal tubulopathies are rare diseases often diagnosed in children, particularly those with autosomal recessive transmission. Although some tubulopathies are diagnosed in adulthood, including recessive diseases with potentially mild presentation (e.g., Gitelman syndrome), slowly progressive dominant diseases (e.g., autosomal dominant tubulointerstitial kidney disease), and diseases with variable severity (e.g., Dent disease), the prevalence of tubulopathies in adults remains mostly unknown. ${ }^{1-3}$ In addition, the clinical presentation may be atypical or insidious, complicating and delaying the diagnosis of tubular dysfunction.

Advances in understanding renal tubular solute transport systems has been achieved through the elucidation of 
monogenic tubular disorders and follow-up studies using cellular and animal models. ${ }^{5}$ In turn, these advances allowed us to improve molecular diagnosis and genetic counselling, as well as to devise therapeutic or preventive measures. ${ }^{6}$ The introduction of high-throughput gene panel sequencing makes the analysis of several genes at the same time possible for genetically heterogeneous diseases and allows for accurate diagnosis in patients with atypical presentations. ${ }^{7}$

We recently published the experience of 3 European centers using a next-generation sequencing panel of 37 known genes to assess children with suspected tubulopathies and confirmed genetic disease in two-thirds of those tested. ${ }^{8}$ In this article, we describe our results in a large adult population using the same panel as well as an improved second version containing 9 additional genes.

\section{RESULTS}

\section{Patients}

The clinical diagnoses of the adult cohort of 1033 patients comprise 20 different tubulopathies and familial hypercalcemia hypocalciuria (FHH) (Table 1). Four diagnoses correspond to $75 \%$ of the cohort: FHH $(N=289)$; Gitelman syndrome $(G S)(N=272)$; renal hypophosphatemia (RH) $(N=124)$, which includes nephrolithiasis/osteoporosis hypophosphatemic and hereditary hypophosphatemic rickets with hypercalciuria; and distal renal tubular acidosis (dRTA) $(N=86)$. Details of additional suspected diagnoses and their proportion in the cohort are summarized in Table 1.

\section{Overall diagnostic performance: initially suspected and diagnostic revision}

In this cohort, pathogenic or likely pathogenic variants were detected in $26 \%$ of cases (269 of 1033), allowing a genetic confirmation. In $24.5 \%$ of cases (253 of 1033), these variants were directly related to the diagnosis initially suspected. In contrast, in $1.5 \%$ of cases (16 of 1033), the genetic findings corresponded to a different diagnosis, symptoms of which may phenocopy the suspected initial pathology. If we consider only tubulopathies, the revision rate is $2.1 \%$ ( 16 of 744 ). The global performance is summarized in Supplementary Figure S1.

Genetic variants. A total of 275 variants were detected in 26 of the 46 genes included in version 2 of the panel; onethird of these variants were not previously described (Table 2). And $86 \%$ of the variants (236 of 275 ) were classified as class 4 or 5 and 25\% (59 of 236) were not previously described. Table 3 summarizes novel variants classified as class 4 or 5 along with the criteria used for their classification. The variants already reported as well as class 3 variants are summarized by disease and by gene in Supplementary Table S1.

The analysis of 90 relatives from 56 different families allowed confirmation of (i) the genetic diagnosis in 29 affected relatives; (ii) compound heterozygosity in patients with recessive diseases after the analysis of parents or children in 22 families, (iii) confirmation of heterozygous carrier status in 2 cases of Xlinked disease; and exclusion of familial disease in 15 relatives of patients with autosomal dominant disorders.

Table 1 | Clinical and genetic diagnoses in the cohort of 1033 adult subjects

\begin{tabular}{|c|c|c|c|c|}
\hline Clinical diagnoses & Patients, $n$ (\%) & Genetic diagnoses, $n$ (\%) & Diagnosis revision, $n$ & $\begin{array}{l}\text { Global diagnostic rate, } \\
\text { confirmed + revised, \% }\end{array}$ \\
\hline $\mathrm{FHH}$ & $289(28)$ & $36(12.5)$ & 0 & 12.5 \\
\hline GS & $272(26)$ & $117(43)$ & 8 & 46 \\
\hline $\mathrm{RH}$ & $121(12)$ & $22(18.2)$ & 0 & 18.2 \\
\hline $\mathrm{dRTA}^{\mathrm{a}}$ & $86(8)$ & $18(20.9)$ & 3 & 24.4 \\
\hline ADTKD & $47(4.5)$ & $8(17)$ & 1 & 19.1 \\
\hline Dent & $37(3.6)$ & $18(48.6)$ & 1 & 51.4 \\
\hline BS & $30(3)$ & $6(20)$ & 1 & 23.3 \\
\hline $\mathrm{ADH}$ & $34(3)$ & $3(8.8)$ & 0 & 8.8 \\
\hline $\mathrm{PHA} 2$ & $28(2.7)$ & $11(39.3)$ & 0 & 39.3 \\
\hline HOMG & $24(2.3)$ & $2(8)$ & 1 & 12.5 \\
\hline $\mathrm{IH}$ & $15(1.5)$ & $3(20)$ & 1 & 26.7 \\
\hline RG & $15(1.5)$ & 7 (47) & 0 & 47 \\
\hline $\mathrm{NC}$ or $\mathrm{FNL}$ & $10(1)$ & $1(10)$ & 0 & 10 \\
\hline NDI & $3(0.3)$ & $0(0)$ & 0 & 0 \\
\hline PHA1 & $1(0.1)$ & $0(0)$ & 0 & 0 \\
\hline Mixed RTA & $1(0.1)$ & $1(100)$ & 0 & 100 \\
\hline Hypertension ${ }^{b}$ & $2(0.2)$ & 0 & 0 & 0 \\
\hline NSIAD & $4(0.4)$ & 0 & 0 & 0 \\
\hline Fanconi & $2(0.2)$ & 0 & 0 & 0 \\
\hline Hyperuricemia & $2(0.2)$ & 0 & 0 & 0 \\
\hline Hypouricemia & $2(0.2)$ & 0 & 0 & 0 \\
\hline Total & 1033 & $253(24.5)$ & 16 & 26 \\
\hline
\end{tabular}

$\mathrm{ADH}$, autosomal dominant hypocalcemia; ADTKD, autosomal dominant tubulointerstitial kidney disease; BS, Bartter syndrome; Dent, Dent disease; dRTA, distal renal tubular acidosis; Fanconi, renal Fanconi syndrome; FHH, familial hypercalcemic hypocalciuria; FNL, familial nephrolithiasis; GS, Gitelman syndrome; HOMG, hypomagnesemia; IH, infantile hypercalcemia; mixed RTA, mixed renal tubular acidosis; NC, nephrocalcinosis; NDI, nephrogenic diabetes insipidus; NSIAD, nephrogenic syndrome of inappropriate anti-diuresis; PHA 1 (2), pseudohypoaldosteronism type 1 (2); RG, renal glycosuria; RH, renal hypophosphatemia (including hypophosphatemic rickets).

${ }^{a}$ Complete and incomplete.

${ }^{b}$ With hypokalemia or normokalemia. 
Table 2 | Molecular diagnosis

\begin{tabular}{|c|c|c|c|c|c|c|}
\hline \multirow[b]{2}{*}{ Disease (\#MIM) } & \multirow[b]{2}{*}{ Gene } & \multicolumn{3}{|c|}{ Patients } & \multicolumn{2}{|l|}{ Variants $^{\mathrm{a}}$} \\
\hline & & Diagnostic confirmed $^{\mathrm{b}}$ & Not confirmed $^{\mathrm{c}}$ & Total & Previously reported & Novel \\
\hline FHH1 (145980) & CASR & 29 & 2 & 31 & 14 & 16 \\
\hline $\mathrm{FHH} 2$ (145981) & GNA11 & 2 & 1 & 3 & 0 & 3 \\
\hline GS (263800) & $S L C 12 A 3$ & 120 & 23 & 143 & 92 & 18 \\
\hline RH (612286) & SLC34A1 & 2 & 2 & 4 & 2 & 2 \\
\hline$(241530)$ & SLC34A3 ${ }^{@}$ & 16 & 3 & 19 & 13 & 10 \\
\hline (602722) & ATP6V0A4 & 1 & 2 & 3 & 3 & 1 \\
\hline (179800) & $S L C 4 A 1$ & 16 & 0 & 16 & 6 & 2 \\
\hline ADTKD (162000) & $U M O D$ & 8 & 1 & 9 & 3 & 6 \\
\hline Dent1 (300009) & CLCN5 & 15 & 1 & 16 & 13 & 1 \\
\hline Dent2 (300555) & OCRL & 3 & 0 & 3 & 3 & 0 \\
\hline BS1 (601678) & $S L C 12 A 1$ & 3 & 2 & 5 & 1 & 7 \\
\hline$(614492)$ & WNK1 & 1 & 1 & 2 & 0 & 2 \\
\hline$(614491)$ & WNK4 & 1 & 0 & 1 & 1 & 0 \\
\hline FHHNC (248250) & CLDN16 & 1 & 0 & 1 & 1 & 0 \\
\hline HOMG1 (602014) & TRPM6 & 1 & 0 & 1 & 0 & 1 \\
\hline IH1 (143880) & CYP24A1 & 3 & 1 & 4 & 2 & 1 \\
\hline IH2 (616963) & SLC34A1 & 0 & 1 & 1 & 0 & 1 \\
\hline RG (233100) & $S L C 5 A 2$ & 7 & 2 & 9 & 6 & 5 \\
\hline pRTA (604278) & SLC4A4 & 0 & 1 & 1 & 0 & 1 \\
\hline Mixed RTA (259730) & CA2 & 1 & 0 & 1 & 1 & 0 \\
\hline Hypouricemia (220150) & $S L C 22 A 12$ & 0 & 1 & 1 & 0 & 1 \\
\hline Total & 26 genes & 269 & 53 & 322 & 180 & 95 \\
\hline
\end{tabular}

\section{Performance by disease}

The detection rates of mutations by disease entity are shown in Figure 1a.

Familial hypercalcemia hypocalciuria. FHH constitutes the largest part of our cohort $(28 \%$; 289 of 1033 patients). Patients with this diagnosis were mainly included by the Paris center. Among patients with this clinical diagnosis, $12.5 \%$ of cases (36 of 289) were genetically confirmed. Mutations were identified in the CASR gene in $81 \%$ ( 29 of $36 \mathrm{FHH} 1$ ), in the AP2S1 gene in 14\% (5 of $36 \mathrm{FHH} 3$ ), and in the GNA11 gene in $6 \%$ ( 2 of $36 \mathrm{FHH} 2$ ). The available clinical and biochemical characteristics of these patients are summarized in Supplementary Table S2.

Tubulopathies. GS represents the most important clinical diagnosis of the tubulopathies in this cohort $(26 \% ; 272$ of 1033). Among these, $43 \%$ of cases (117 of 272 ) were genetically confirmed-detection of 2 heterozygous class 4 or 5 variants in the SLC12A3 gene.

$\mathrm{RH}$ corresponds to $12 \%$ of the cohort (124 of 1033) with a mutation detection rate of $18 \%$ (22 of 124). Thirteen patients had variants in the SCL34A3 gene: 8 had 1 heterozygous variant, and 5 had 2 heterozygous variants (unfortunately
DNA from relatives was unavailable for segregation studies). Seven patients had heterozygous pathogenic variants in the SLC9A3R1 gene. Finally, 2 patients had heterozygous pathogenic variants in the SLC34A1 gene. Interestingly 1 of patients with clinical and genetic diagnosis of $\mathrm{RH}$ with pathogenic heterozygous variant SCL34A3 has also a class 5 variant in the SLC9A3R1 gene, raising the possibility of digenism.

The third most common tubulopathy in the cohort is dRTA, representing $8 \%$ of the patients ( 86 of 1033). Out of the 86 patients with this diagnosis, 18 were genetically confirmed $(21 \%)$. SLC4A1 was implicated in 15 patients (83\%), ATP6V1B1 in 2 patients, and ATP6V0A4 in 1 patient.

Autosomal dominant tubulointerstitial kidney disease related to UMOD represents $4.5 \%$ of the cohort (47 of 1033), and $17 \%$ of these patients ( 8 of 47 ) had genetic confirmation. Dent disease represents $3.6 \%$ of the cohort (37 of 1033), with a rate of genetic confirmation of $49 \%$ (18 of 37). Fifteen patients carried CLCN5 variants (Dent 1), and 3 patients carried OCRL variants (Dent 2). Bartter syndrome (BS) and autosomal dominant hypocalcemia each represent $3 \%$ of the cohort (31 and 34 of 1033) with a rate of genetic confirmation of 20\% (6 of 31) and 9\% (3 of 34), respectively. 
Table 3 | Novel class 4 and 5 variants detected in this study and their classification according to ACMG

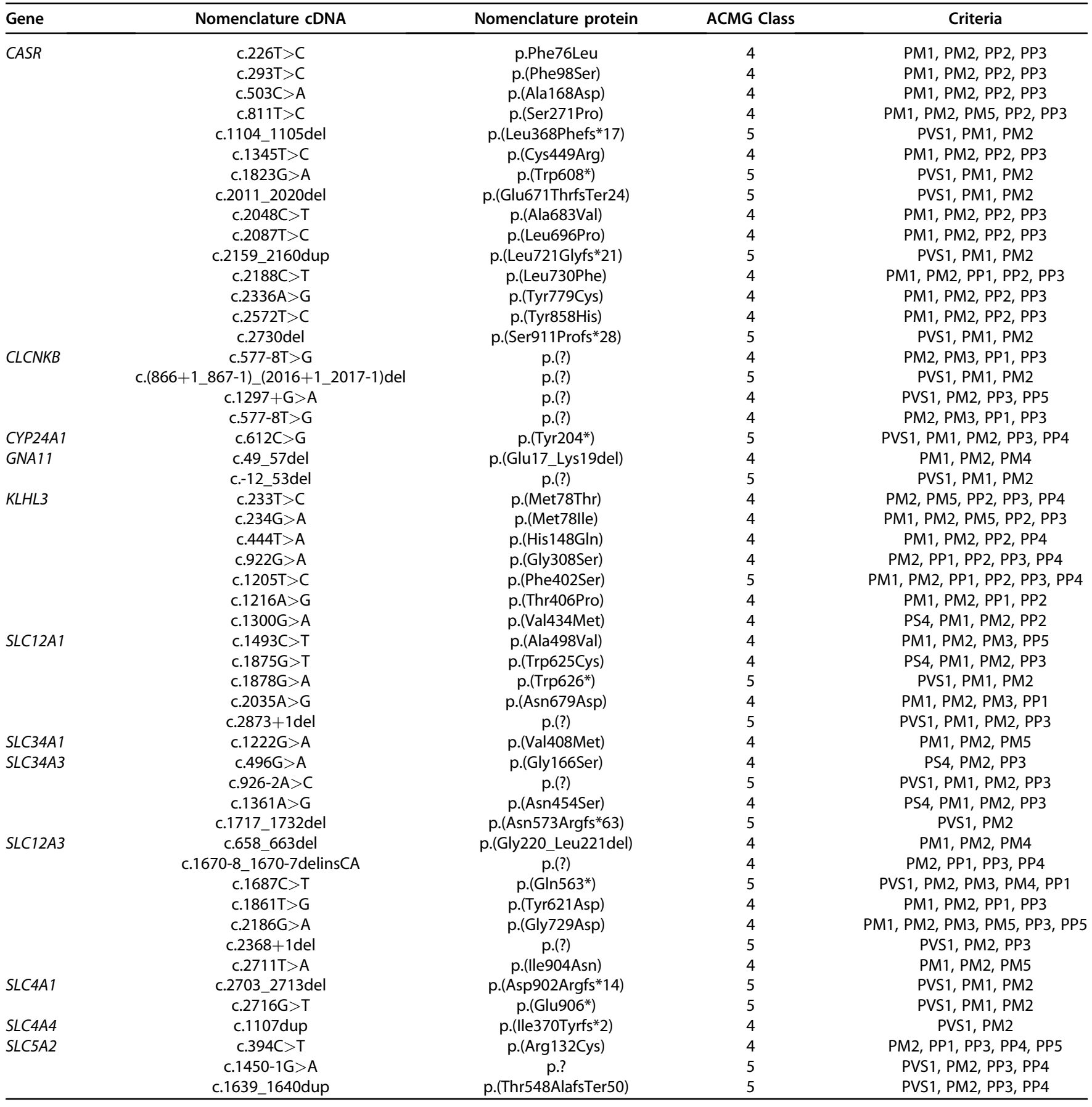

(Continued on next page)

The following diagnoses each represent less than $3 \%$ of the cohort and have a rate of genetic confirmation between $18 \%$ and 50\% (Table 1): Pseudohypoaldosteronism type 2 (PHA2), hypomagnesemia (HOMG), infantile hypercalcemia (IH), and renal glycosuria. Finally, 1 patient with clinical diagnosis of mixed RTA had a genetic confirmation (homozygous class 4 variant in the CA2 gene).

Other clinical diagnoses, for which no genetic confirmation was made included: PHA1, nephrocalcinosis (NC), hypertension, nephrogenic diabetes insipidus, nephrogenic syndrome of inappropriate antidiuresis, Fanconi syndrome, hypouricemia, and hyperuricemia (Table 1).

Patients with variants of unknown significance. Thirty variations detected in 16 different genes in 40 patients were classed as class 3 or variants of unknown significance. They are described by disease and by gene in Supplementary Table S1. In 10 patients with clinical BS or GS, the class 3 variant was associated with a class 4 or 5 variant in the same gene. 
Table 3 (Continued) Novel class 4 and 5 variants detected in this study and their classification according to ACMG

\begin{tabular}{lcccc}
\hline Gene & Nomenclature cDNA & Nomenclature protein & ACMG Class & Criteria \\
\hline TRPM6 & c.278A $>$ G & p.(Asp93Gly) & 4 & PM2, PM3, PP1, PP4 \\
UMOD & c.179G $>$ C & p.(Gly60Ala) & 4 & PM1, PM2, PP3, PP4 \\
& c.478G $>$ C & p.(Asp160His) & 4 & PM1, PM2, PP3, PP4 \\
& c.692T $>$ C & p.(Leu231Pro) & 4 & PM1, PM2, PP3, PP4 \\
& c.851T $>$ C & p.(Leu284Pro) & 4 & PM1, PM2, PP3, PP4 \\
WNK1 & c.274T $>$ C & p.(Cys92Arg) & 4 & PM1, PM2, PM (segregation) \\
\hline
\end{tabular}

American College of Medical Genetics (ACMG) criteria description: PM, moderate evidence of pathogenicity; PM1, located in a mutational hot spot or critical and well-established functional domain without benign variation; PM2, absent from controls (or at extremely low frequency if recessive) in gnomAD database (https://gnomad.broadinstitute.org/); PM3, detected in trans with a pathogenic variant (the phase was determined); PM4, protein length changes due to in-frame deletions or insertions in a nonrepeat region or stop-loss variants; PM5, novel missense change at an amino acid residue where a different missense change determined to be pathogenic has been seen before; PP, supporting evidence of pathogenicity; PP1, cosegregation with disease in multiple affected family members in a gene definitively known to cause the disease; PP2, missense variant in a gene that has a low rate of benign missense variation and in which missense variants; PP3, multiple lines of computational evidence support a deleterious effect on the gene or gene product; PP4, patient's phenotype or family history is highly specific for a disease with a single genetic etiology; PP5, reputable source recently reports variant as pathogenic but the evidence is not available to the laboratory to perform an independent evaluation; PS, strong evidence of pathogenicity; PS1, same amino acid change as a previously established pathogenic variant regardless of nucleotide change; PS3, well-established in vitro or in vivo functional studies supportive of a damaging effect on the gene or gene product; PS4, the prevalence of the variant in affected individuals is significantly increased compared with the prevalence in controls; PVS, very strong evidence of pathogenicity; PVS1, null variant (nonsense, frameshift, canonical \pm 1 or 2 splice sites, initiation codon, single or multi-exon deletion) in a gene where loss of function is a known mechanism of disease.

Genetic revision of the clinical diagnosis. In 16 cases, the analysis of other genes present in the panel allowed a revision of the diagnosis. Table 4 summarizes these cases.

First, one-half of these cases correspond to the known overlap of phenotypes of GS and classic BS that turned out to be genetically either BS type 3 or GS.

Second, 1 patient with clinical diagnosis of autosomal dominant tubulointerstitial kidney disease had a genetic diagnosis of BS type 1 (patient B26). This patient had a diagnosis of chronic kidney disease at the age of 32 years, in absence of other manifestations except polyuria; she had hyperuricemia and normal level of potassium. Her oldest sister had gouty arthritis before the age of 30 years. The patient and her younger sister had nephrocalcinosis. DNA samples were not available to test family segregation. a

Adult tubulopathy cases

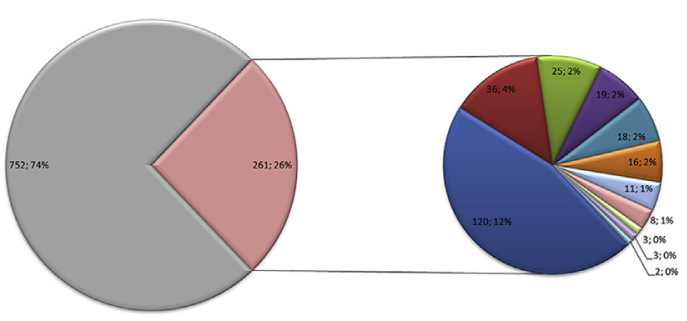

$\because \mathrm{GS}$

$\square \mathrm{FHH}$

$\square \mathrm{RH}$

a dRTA

$\square$ Dent

$\square \mathrm{BS}$

$\triangle \mathrm{PHA} 2$

$\square$ ADTKD

$\square \mathrm{IH}$

$\triangle \mathrm{ADH}$

$\square \mathrm{HOMG}$

$\square$ Negative b

Child tubulopathy cases
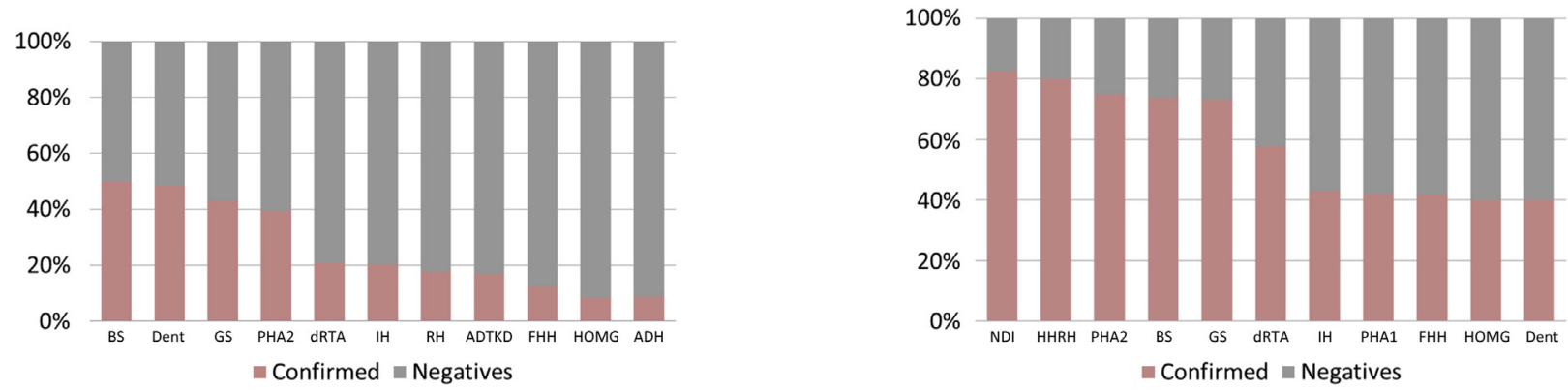

Figure 1 | Pie charts $(n ; \%)$ and bar graphs comparing detection rates, distribution of genetically confirmed pathologies, and confirmation rate by disease in (a) adults and (b) children. $A D H$, autosomal dominant hypocalcemia; ADTKD, autosomal dominant tubulointerstitial kidney disease; BS, Bartter syndrome; Dent, Dent disease; dRTA, distal renal tubular acidosis; East S, epilepsy, ataxia, sensorineural deafness, and tubulopathy; $\mathrm{FHH}$, familial hypercalcemia hypocalciuria; GS, Gitelman syndrome; HHRH, hereditary hypophosphatemic rickets with hypercalciuria; HOMG, hypomagnesemia; IH, infantile hypercalcemia; NDI, nephrogenic diabetes insipidus; PHA1 (2), pseudohypoaldosteronism type 1 (2); pRTA, proximal renal tubular acidosis; RH, renal hypophosphatemia. 

Table 4 | Index cases with genetic revision of the clinical
diagnosis

\begin{tabular}{lcc}
\hline Patient & Clinical diagnosis & Genetic diagnosis \\
\hline L81 & Gitelman & Bartter 3 \\
L117 & Gitelman & Bartter 3 \\
L123 & Gitelman & Bartter 3 \\
P56 & Gitelman & Bartter 3 \\
P104 & Gitelman & Bartter 3 \\
P116 & Gitelman & Bartter 3 \\
P608 & Gitelman & Bartter 3 \\
L85 & Bartter 3 & Gitelman \\
B26 & ADTKD & Bartter 1 \\
L84 & Incomplete dRTA & Bartter 2 \\
L150 & Incomplete dRTA & Gitelman \\
P6 & Incomplete dRTA & Renal hypophosphatemia \\
P113 & Hypomagnesemia & Gitelman \\
P179 & Dent & dRTA \\
B61 & Familial nephrolithiasis & Renal hypophosphatemia \\
P582 & Infantile hypercalcemia & Renal hypophosphatemia \\
\hline ADTKD, autosomal dominant tubulointerstitial kidney disease; dRTA, distal renal \\
tubular acidosis.
\end{tabular}

Third, 3 cases with clinical diagnosis of incomplete dRTA were genetically diagnosed as type 2 BS (patient L84), GS (patient L150), and RH (patient P6). Patient L84 had hypokalemia and nephrocalcinosis diagnosed age 35 years. She was referred to nephrology 3 years later and is on potassium supplements and amiloride. Her parents were first cousins and she had a strong family history of kidney stones. Plasma bicarbonate at presentation was $26 \mathrm{mmol} / \mathrm{l}$, and the initial clinical diagnosis was of incomplete dRTA. Patient L150 is from Turkish Cyprus, who had severe acidosis (bicarbonate $12 \mathrm{mmol} / \mathrm{l}$ ), hypokalemia (potassium 2.7-3 mmol/l), normal plasma magnesium and chloride, and a urinary $\mathrm{pH}$ of 8 . He was on potassium supplements and spironolactone at the time of genotyping. Patient P6 had a history of kidney stones and hypocitraturia; an acid load test showed an inappropriate urinary $\mathrm{pH}$ (5.8) and an abnormal ammonia excretion establishing the diagnosis of incomplete dRTA, nevertheless a borderline phosphate concentration was also observed; in this patient 2 heterozygous variants in SLC34A3 were detected.

Fourth, the patient (P113) with renal HOMG who had a final diagnosis of GS presented with severe HOMG (0.25 $\mathrm{mmol} / \mathrm{l})$ and borderline hypokalemia $(3.4-3.9 \mathrm{mmol} / \mathrm{l})$; the hypokalemia was initially considered secondary to HOMG.

Fifth, in 1 patient (P179) with clinical diagnosis of Dent disease, the genetic diagnosis was an autosomally dominant dRTA. This patient with a history of stone disease was given a diagnosis of medullary sponge kidney disease at 20 years old; an evaluation 3 years later showed proximal tubulopathy and chronic kidney disease: renal hypophosphatemia, hyperchloremic metabolic acidosis, hypocitraturia, proteinuria $(1.41 \mathrm{~g} / \mathrm{d}$ with moderate albuminuria $143 \mathrm{mg} / \mathrm{d})$ and inulin clearance of $58 \mathrm{ml} / \mathrm{min}$ per $1.73 \mathrm{~m}^{2}$. No variants were detected in genes responsible for Dent disease or renal Fanconi syndrome. Taking into account the presence of stone disease and hypocitraturia, we looked for variants in genes responsible of dRTA and detected a known class 5 variant in the SLC4A1 gene.

Finally, 2 cases (B61 and P582) with clinical diagnosis of $\mathrm{IH}$ and nephrolithiasis (NL) had heterozygous variants in the SLC34A3 gene.

\section{DISCUSSION}

Hereditary tubulopathies are less frequently diagnosed in adults. However, the establishment of a precise diagnosis is important to guide care, survey, and potentially prevent chronic complications as well as for genetic counselling. In this paper, we describe the analysis of a large multicenter cohort of adult patients with a panel of 46 genes involved in tubulopathies or in $\mathrm{FHH}$, which yielded an overall genetic confirmation in $26 \%$ of cases after the detection of pathogenic variants in 26 different genes.

Twenty-eight percent of cases of this cohort have a clinical diagnosis of $\mathrm{FHH}$, which is not primarily a tubulopathy. The genes responsible for this disease have been included due to the role of the corresponding proteins in tubular calcium reabsorption. In addition, patients with this diagnosis were mainly included by the Paris center. With the exclusion of this disease, the rate of genetic confirmation increases to $29 \%$ (216 of 744), which is approximately one-half of the rate we observed previously in children $(64 \%) .{ }^{8}$ This percentage remains unchanged when genes presented only in the second panel version are excluded (see Supplementary Table S3 for panel composition).

A recent study analyzed a panel of 30 genes that cause $\mathrm{NC} /$ NL in a cohort of children and adults showing that the detection rate of monogenic causes in adults was one-half that seen in children ( $11.4 \%$ vs. $20.8 \%)^{9}$

Several features may explain the lower rate observed in adults. First, autosomal recessive tubulopathies are mainly diagnosed in children. Accordingly, Halbritter et al. ${ }^{9}$ in their study of monogenic causes of NC/NL found that recessive causes were diagnosed more frequently in childhood, whereas dominant disease was diagnosed more commonly in adulthood. Nevertheless, in our cohort autosomal recessive diseases were confirmed in 153 of 269 patients (57\%), primarily in patients with GS $(n=120)$ and BS $(n=16)$.

Second, the presence of comorbidities and polypharmacy in cohorts of adult patients can make interpretation of the phenotype more difficult (especially when the phenotype involves chronic kidney disease) or complicate the interpretation of urinary chemistry. Also acquired tubular dysfunction (e.g., autoimmune dRTA, renal Fanconi syndrome caused by drug or environmental toxicity) is much more common in adults, and genetic testing may be an important part of investigation for these patients. ${ }^{10-12}$

Third, a significant number of tests are performed as exclusion diagnosis. This is especially true for patients with clinical diagnosis of $\mathrm{FHH}$, for which we have a low diagnosis rate $(12.5 \%)$. FHH could have a very similar clinical presentation to primary hyperparathyroidism, and, despite proposed scores and algorithms, it remains difficult to have a 
precise clinical diagnosis. ${ }^{13}$ It is therefore recommended in these cases to exclude a diagnosis of FHH before committing to parathyroidectomy, which is inappropriate in $\mathrm{FHH}^{14}$ We were able to analyze the data of 86 patients for whom a genetic diagnosis of FHH was excluded and follow-up information was available. Thirty-five patients $(41 \%)$ were parathyroidectomized and 31\% of them (11 of 35) normalized their serum calcium level postoperatively while the remaining 24 patients had persistent hypercalcemia. This percentage is higher than the usual rate of unsuccessful parathyroidectomy in primary hyperparathyroidism $(5 \%-$ $10 \%)$. The diagnosis of $\mathrm{FHH}$ is not completely excluded in this population because there are probably other genes responsible for this disease. ${ }^{15}$ This highlights the importance of pursuing further research genetic investigations to identify other genes responsible for these rare phenotypes. Concerning the clinical presentation of different types of $\mathrm{FHH}$, although there are few cases of $\mathrm{FHH} 2$ and $\mathrm{FHH} 3$ in this cohort; analysis of clinical data (Supplementary Table S2) shows similar results to results described in the literature, that is, higher hypercalcemia in patients with $\mathrm{FHH} 3$. $^{16,17}$

GS was the most common tubulopathy in adults with a high molecular confirmation rate (46\%) (Figure 1). The main symptoms of GS are rather nonspecific (cramps, poorly defined discomfort, fatigue) and they evolve slowly, resulting in a late or incidental presentation. While the biochemical phenotype is typically well characterized, explaining the high level of genetic diagnosis, patient L150, presenting with acidosis, demonstrates that it can be surprisingly variable. Notably, in $55 \%$ of patients with available clinical information (42 of 76), the genetic test was performed to exclude the diagnosis (e.g., in patients with eating disorders or surreptitious diuretic abuse and questionable biological data) and as expected no variants were detected. ${ }^{18}$

In contrast with GS, BS, the most common tubulopathy in children, is rarely diagnosed in adulthood. BS was confirmed in 16 patients. As expected, most of them $(n=12)$ had BS type 3. Three patients (P103, B26, B46) had genetic diagnosis of BS type 1 and 1 patient (L84) had BS type 2. Patients B26 and L84 correspond to revised diagnosis. Patient P103 had a diagnosis of hypokalemia made when she was 30 years old on routine examination during her first pregnancy; later evaluation revealed alkalosis, high renin level, and hypercalciuria. She harbored 2 missense class 4 heterozygous variants in SLC12A1 located in trans (her son is heterozygous for 1 of 2 variants). The presentation in the woman B46 was quite similar with reduced serum potassium concentration at age of 35 years with mild HOMG and hypercalciuria. The patient was heterozygous for a class 4 missense change and a complete deletion of exon 16. This rearrangement was expected to lead to a frameshifted transcript. Whereas BS type 1 is in general associated with polyhydramnios and severe neonatal manifestations, there is a description of late-onset manifestations related with partial loss of function of the mutant. ${ }^{19} \mathrm{~A}$ similar situation could explain the late onset in these patients, which needs confirmation by in vitro studies.
Among patients with clinical BS and GS, 10 cases harbored 2 variants, but 1 of them was considered as class 3 ; for these variants there are no criteria for benignity and the confirmation that they are located in trans after familial segregation studies will allow us to reclassify them as class 4 , increasing the confirmation rate.

$\mathrm{NL} / \mathrm{NC}$ is a common clinical manifestation in adult patients. The main monogenic causes of NL/NC are Dent disease, RH, complete and incomplete dRTA, and IH, representing $17 \%$ of cases with genetic confirmation in our cohort. For patients with clinical diagnosis of Dent disease, we had a higher genetic confirmation rate in adults than in children ( $49 \%$ vs. $40 \%)$. This may be explained by the incomplete renal Fanconi syndrome that they have as infants, which is easy to miss clinically, and the fact that nephrocalcinosis and renal failure may only become evident during adult life. Causative genes were CLCN5 in $83 \%$ of cases and OCRL in $17 \%$, which is similar to that described in the literature. ${ }^{20}$ The contribution of molecular diagnosis for these patients is crucial, given the progression to end-stage kidney disease (typically in the fourth or fifth decade of life), allowing genetic counselling and detection of heterozygous female carriers. For RH and dRTA, we observed a relatively lower detection rate that interestingly is similar to the rate described by Halbritter et al. ${ }^{9}$ in adults. This lower detection rate may be explained by the multifactorial origin of $\mathrm{NL} / \mathrm{NC}$, but we cannot exclude variants not detected by our method (i.e., deep intronic variants), known genes not present in our panel (i.e., SLC7A9, ADCY10), or a supplementary genetic heterogeneity. ${ }^{9,21}$ In 5 patients with NL, we detected 2 pathogenic heterozygous variants of the SLC34A3 gene; unfortunately DNA from relatives was unavailable to confirm that they are biallelic; 2 of these 5 patients presented with osteopenia and 2 others with nephrocalcinosis. Finally, type $1 \mathrm{IH}$ was confirmed in 3 patients. Previous reports and this data confirm that IH can also be an adult disease. Heterozygous patients can have hypercalciuria and NL as well as high calcitriol levels. ${ }^{22,23}$ In 1 of our index cases (P580) only 1 heterozygous class 5 variant was detected. Although we cannot exclude the presence of a second variant in a nonanalyzed region of the gene; this observation rises also the possibility of clinical manifestation in heterozygous patients.

In PHA2, we found a high rate of confirmed diagnoses (39\%), mainly explained by pathogenic variants in KLHL3. The biochemical phenotype of PHA2 (hypertension, hyperkalemia, and metabolic acidosis) is quite specific, which may explain this high rate. KLHL3 molecular abnormalities explain a high proportion of patients with this disease in the cases described in the literature. ${ }^{24}$

For the patients with HOMG suspected of genetic origin, 2 patients had HOMG related to TRPM6 mutations (HOMG1). This syndrome is usually diagnosed during the first year of life, with convulsions associated with severe hypocalcemia. ${ }^{25}$ Patient P555 from a consanguineous family had a diagnosis of HOMG secondary to intestinal malabsorption at 32 years old; she had secondary hypoparathyroidism and no 
neurological manifestations, again demonstrating the spectrum of clinical severity.

The rate of discrepancy between clinical and genetic diagnosis is relatively low (2.1\%), which underlines the diagnostic accuracy of the referring clinicians. A total of 16 patients had their initial clinical diagnosis revised by the panel analysis. As in children, the revision was rarely substantial and mostly concerns salt-losing tubulopathies or diseases associated with NC/NL or a combination of these. Several tubulopathies present with either hypokalaemia or hypercalciuria or both and thus can constitute phenocopies of these findings (Figure 2).

The most common revision was from GS to BS type 3 or vice versa in 8 cases, which was expected taking into account the well-known phenotypic overlap between these 2 syndromes. Three cases with clinical diagnosis of incomplete dRTA were found to type 2 BS, GS, and RH (L84, L150, P6), respectively. Two cases (B61 and $\mathrm{P} 582$ ) with clinical diagnosis of IH and NL had heterozygous variants in the SLC34A3 gene. This interesting observation shows that patients with absorptive hypercalciuria due to increased calcitriol as in IH or renal hypophosphatemia can have overlapping phenotypes and clinical and biological manifestations heterozygously. ${ }^{22,23,26}$ For patient B26 with genetic diagnosis of BS type 1 , the clinical diagnosis of autosomal dominant tubulointerstitial kidney disease could have been excluded due to the presence of nephrocalcinois. ${ }^{2}$ Finally, in 1 patient (P179) with clinical diagnosis of Dent disease the genetic diagnosis was an autosomally dominant dRTA.

In conclusion, this work demonstrates that a genetic cause can be demonstrated in 1 of 3 adult patients presenting with a clinical suspicion of tubulopathy. This rate of detection is one-half of that observed in children. The establishment of a genetic diagnosis is crucial for the patient to guide care, survey, and prevent chronic complications, as well as for genetic counselling. These results enhance our understanding of complex phenotypes and enrich the database of causal variants associated with clinically defined tubulopathies.

\section{METHODS}

\section{Patients}

A total of 1033 adult index cases with a clinical diagnosis of tubular dysfunction made after the age of 18 years and for which the implicated gene(s) are present in the panel were included and analyzed from 2014 to 2016 . The clinical diagnosis was performed by physicians belonging to the network of 3 expert centers. Of these, 139 samples were analyzed in Brussels, 171 in London, and 723 in Paris. The last center has a large recruitment of patients with hypercalcemia of parathyroid origin, explaining the inclusion of a large number of patients with suspected $\mathrm{FHH}$, which is not strictly speaking a tubulopathy. In addition, 90 relatives belonging to 56 families in whom a pathogenic variant was detected in the proband were analyzed; these included 29 affected individuals. Informed consent for genetic testing was obtained by the respective treating physician after approval by the respective institutional review boards, in accordance with the Declaration of Helsinki.

\section{Gene amplification, sequencing, and bioinformatic analysis}

This was performed as described previously. ${ }^{8}$ Briefly, genomic DNA was isolated from white blood cells using standard procedures, followed by massive parallel sequencing using the versions 1 and 2 of a tubulopathies panel designed by the work package tubulopathies of the European consortium EURenOmics. The first version was previously described in our paediatric cohort. ${ }^{7}$ The second version includes 9 additional genes. Supplementary Table S3 compares the composition of these 2 versions.
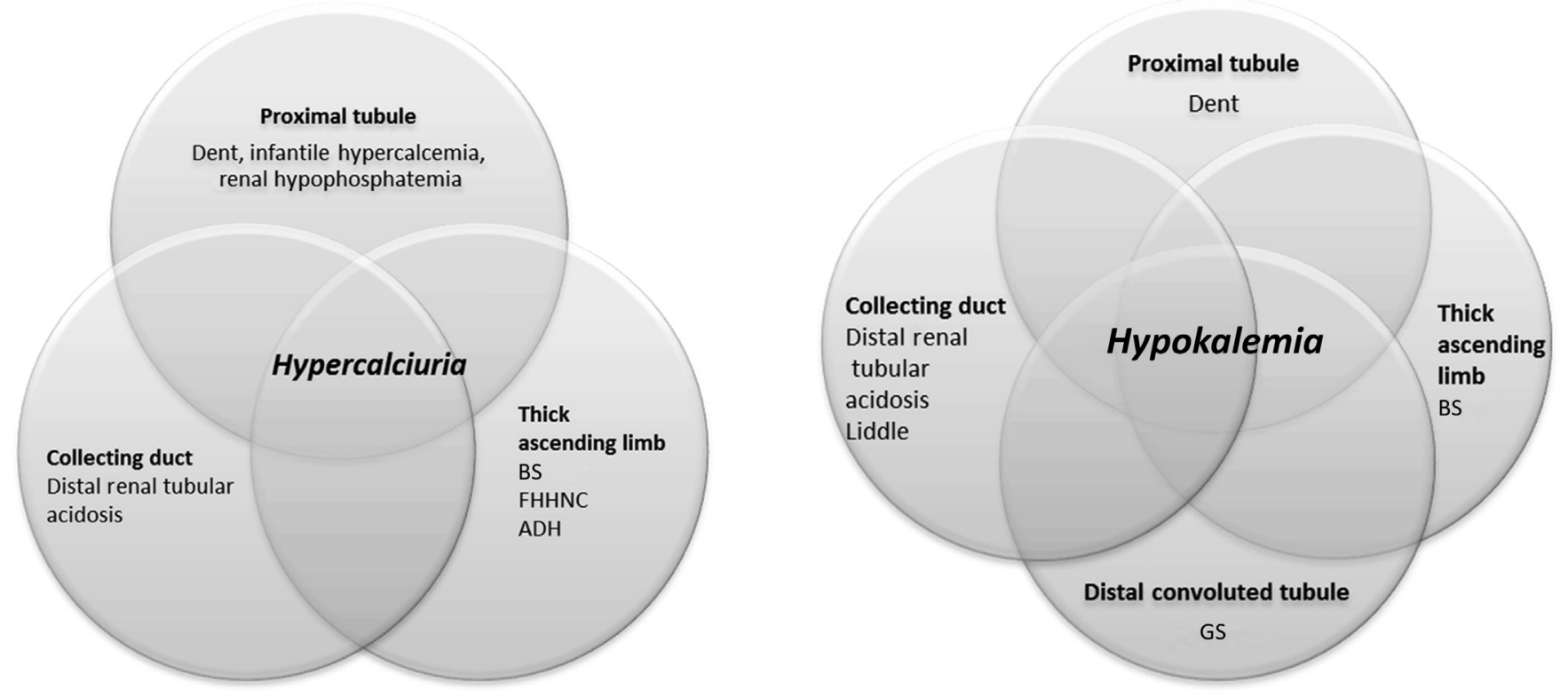

Figure 2| Tubulopathies phenocoping 2 frequent clinical presentations in the adult cohort. ADH, autosomal dominant hypocalcemia; BS, Bartter syndrome; Dent, Dent disease; FHHNC, familial hypomagnesemia with hypercalciuria and nephrocalcinosis; GS, Gitelman syndrome; Liddle, Liddle syndrome. 
The depth for each region of interest was at least $30 \times$, except for exon 1 of OCRL and WNK1, analysis of which was completed by Sanger sequencing in those with suspected disease related to these 2 genes and with no other causative mutation identified. Regions with coverage lower than $30 \mathrm{X}$ were sequenced by Sanger. Library preparation and bioinformatics analysis was performed according to the techniques and pipelines routinely used by each laboratory (Supplementary Methods). The variants were classified according to the guidelines published by the American College of Medical Genetics $2015 .{ }^{27}$ Variants of interest were verified by Sanger sequencing.

The 3 centers are accredited laboratories proposing genetic diagnostic tests referenced in Orphanet (https://www.orpha.net/ consor/cgi-bin/index.php?lng=EN or https://ukgtn.nhs.uk/find-atest/search-by-laboratory/laboratory/london-north-east-rgc-gosh-43/).

\section{DISCLOSURE}

AR and JDF are employees of Multiplicom, provider of the kits used for amplification of the 46 genes. All the other authors declared no competing interests.

\section{ACKNOWLEDGMENTS}

Funding for this study was kindly provided by the European Union, FP7 (grant agreement 2012-305608 "European Consortium for HighThroughput Research in Rare Kidney Diseases (EURenOmics)"; by the British Kidney Patient Association; and by the clinical research priority program Rare Disease Initiative Zurich of the University of Zurich and the project grant 31003A_169850 of the Swiss National Science Foundation.

\section{SUPPLEMENTARY MATERIAL}

Figure S1. Flow diagram of global performance.

Table S1. American College of Medical Genetics classification of all variants detected in this study summarized by disease and by gene. Table S2. Phenotype-genotype correlation in patients with genetically confirmed FHH.

Table S3. Panel composition of versions 1 and 2 of the tubulopathies panel.

\section{Supplementary Methods.}

Supplementary material is linked to the online version of the paper at www.kidney-international.org.

\section{REFERENCES}

1. Blanchard A, Bockenhauer D, Bolignano D, et al. Gitelman syndrome: consensus and guidance from a Kidney Disease: Improving Global Outcomes (KDIGO) Controversies Conference. Kidney Int. 2017;91:24-33.

2. Eckardt KU, Alper SL, Antignac C, et al. Autosomal dominant tubulointerstitial kidney disease: diagnosis, classification, and management-A KDIGO consensus report. Kidney Int. 2015;88:676-683.

3. Blanchard A, Curis E, Guyon-Roger T, et al. Observations of a large Dent disease cohort. Kidney Int. 2016;90:430-439.

4. Khosravi M, Walsh SB. The long-term complications of the inherited tubulopathies: an adult perspective. Pediatr Nephrol. 2015;30:385-395.

5. Van der Wijs J, Belge H, Bindels RJM, Devuyst O. Learning physiology from inherited kidney disorders. Physiol Rev. 2019;99:1575-1653.

6. Devuyst O, Knoers NV, Remuzzi G, et al. for the Board of the Working Group for Inherited Kidney Diseases of the European Renal Association and European Dialysis and Transplantation Association. Rare inherited kidney diseases: challenges, opportunities, and perspectives. Lancet. 2014;383:1844-1859.

7. Groopman EE, Rasouly HM, Gharavi AG. Genomic medicine for kidney disease. Nat Rev Nephrol. 2018;14:83-104.

8. Ashton EJ, Legrand A, Benoit $V$, et al. Simultaneous sequencing of 37 genes identified causative mutations in the majority of children with renal tubulopathies. Kidney Int. 2018;93:961-967.

9. Halbritter J, Baum M, Hynes AM, et al. Fourteen monogenic genes account for $15 \%$ of nephrolithiasis/nephrocalcinosis. J Am Soc Nephrol. 2015;26:543-551.

10. Hall AM, Bass P, Unwin RJ. Drug-induced renal Fanconi syndrome. QJM. 2014;107:261-269.

11. Luo J, Huo YW, Wang JW, Guo H. High-risk indicators of renal involvement in primary Sjogren's syndrome: a clinical study of 1002 cases. J Immunol Res. 2019;17:3952392.

12. Evans R, Zdebik A, Ciurtin C, Walsh SB. Renal involvement in primary Sjogren's syndrome. Rheumatology (Oxford). 2015;54:1541-1548.

13. Bertocchio JP, Tafflet M, Koumakis E, et al. Pro-FHH: a risk equation to facilitate the diagnosis of parathyroid-related hypercalcemia. J Clin Endocrinol Metab. 2018;103:2534-2542.

14. Khan AA, Hanley DA, Rizzoli R, et al. Primary hyperparathyroidism: review and recommendations on evaluation, diagnosis, and management. A Canadian and international consensus. Osteoporos Int. 2017;28:1-19.

15. Cormier C. Genetic hypercalcemia. Joint Bone Spine. 2018;86:459-466.

16. Hannan FM, Babinsky VN, Thakker RV. Disorders of the calcium-sensing receptor and partner proteins: insights into the molecular basis of calcium homeostasis. J Mol Endocrinol. 2016;57:R127-R142.

17. Vargas-Poussou R, Mansour-Hendili L, Baron S, et al. Familial hypocalciuric hypercalcemia types 1 and 3 and primary hyperparathyroidism: similarities and differences. J Clin Endocrinol Metab. 2016;101:2185-2195.

18. Wu KL, Cheng $\mathrm{CJ}$, Sung $\mathrm{CC}$, et al. Identification of the causes for chronic hypokalemia: importance of urinary sodium and chloride excretion. Am $J$ Med. 2017;130:846-855.

19. Pressler CA, Heinzinger J, Jeck N, et al. Late-onset manifestation of antenatal Bartter syndrome as a result of residual function of the mutated renal $\mathrm{Na}+$ K+-2Cl- co-transporter. J Am Soc Nephrol. 2006;17:2136-2142.

20. Devuyst O, Thakker RV. Dent's disease. Orphanet J Rare Dis. 2010;5:28.

21. Courbebaisse M, Prot-Bertoye C, Bertocchio JP, et al. [Nephrolithiasis of adult: From mechanisms to preventive medical treatment]. Rev Med Interne. 2017:38:44-52.

22. Colussi G, Ganon L, Penco S, et al. Chronic hypercalcaemia from inactivating mutations of vitamin D 24-hydroxylase (CYP24A1): implications for mineral metabolism changes in chronic renal failure. Nephrol Dial Transplant. 2014;29:636-643.

23. Molin A, Baudoin R, Kaufmann M, et al. CYP24A1 Mutations in a Cohort of Hypercalcemic Patients: Evidence for a Recessive Trait. J Clin Endocrinol Metab. 2015;100:E1343-E1352.

24. Ellison DH. Pseudohypoaldosteronism type II. In: Adam MP, Ardinger HH, Pagon RA, et al., eds. GeneReviews. Seattle, WA: University of Washington; 2011.

25. Schlingmann KP, Sassen MC, Weber S, et al. Novel TRPM6 mutations in 21 families with primary hypomagnesemia and secondary hypocalcemia. J Am Soc Nephrol. 2005;16:3061-3069.

26. Dasgupta D, Wee MJ, Reyes M, et al. Mutations in SLC34A3/NPT2C are associated with kidney stones and nephrocalcinosis. J Am Soc Nephrol. 2014;25:2366-2375.

27. Richards S, Aziz N, Bale S, et al. for the ACMG Laboratory Quality Assurance Committee. Standards and guidelines for the interpretation of sequence variants: a joint consensus recommendation of the American College of Medical Genetics and Genomics and the Association for Molecular Pathology. Genet Med. 2015;17:405-424. 